\title{
BMJ Open Acupuncture for insomnia with short sleep duration: protocol for a randomised controlled trial
}

\author{
Cong Wang (D) , ${ }^{1}$ Wen-jia Yang, ${ }^{1}$ Xin-tong $\mathrm{Yu}^{2}$ Cong Fu, ${ }^{1}$ Jin-jin Li, ${ }^{1}$ Jing Wang, ${ }^{1}$ \\ Wen-lin Xu, ${ }^{1}$ Yi-xin Zheng, ${ }^{1}$ Xin-yu Chen, ${ }^{3}$ Yun-fei Chen ${ }^{1}$
}

To cite: Wang C, Yang W, Yu X, et al. Acupuncture for insomnia with short sleep duration: protocol for a randomised controlled trial. BMJ Open 2020;10:e033731. doi:10.1136/ bmjopen-2019-033731

- Prepublication history for this paper is available online To view these files, please visit the journal online (http://dx.doi. org/10.1136/bmjopen-2019033731).

Received 19 August 2019 Revised 04 November 2019 Accepted 06 January 2020

Check for updates

(C) Author(s) (or their employer(s)) 2020. Re-use permitted under CC BY-NC. No commercial re-use. See rights and permissions. Published by BMJ.

${ }^{1}$ Department of Acupuncture and Moxibustion, Yueyang Hospital of Integrated Traditional Chinese and Western Medicine, Shanghai University of Traditional Chinese Medicine, Shanghai, China

${ }^{2}$ Laboratory Center of Medicine, Yueyang Hospital of Integrated Traditional Chinese and Western Medicine, Shanghai University of Traditional Chinese Medicine, Shanghai, China

${ }^{3}$ Beijing University of Chinese Medicine Dongfang College, Beijing, China

Correspondence to Professor Yun-fei Chen; icyf1968@163.com

\section{ABSTRACT}

Introduction Insomnia with short sleep duration has a more serious negative impact on patient health. The existing literature suggests that medication therapy is more effective for this phenotype of insomnia compared with cognitive-behavioural therapy. However, the potential side effects of hypnotic medications hinder their clinical application. Acupuncture has been widely used in the treatment of insomnia, but it remains unclear whether it has therapeutic efficacy for insomnia with short sleep duration. The purpose of this trial is to evaluate the efficacy and safety of acupuncture for insomnia with short sleep duration.

Methods and analysis This study is designed as a randomised, single-centre, single-blinded, placebo acupuncture controlled trial involving 152 participants. Eligible patients will be divided into two groups according to the objective total sleep time: insomnia with normal sleep duration group and insomnia with short sleep duration group. Then, patients in each group will be randomly assigned to two subgroups, the treatment group (acupuncture) and the control group (placebo acupuncture), in a 1:1 ratio with 38 subjects in each subgroup. The primary outcome is the Pittsburgh Sleep Quality Index and the Insomnia Severity Index. Secondary outcomes are actigraphy, the Beck Anxiety Inventory, the Beck Depression Inventory and the Fatigue Severity Scale. All adverse effects will be assessed by the Treatment Emergent Symptom Scale. Outcomes will be evaluated at baseline, post treatment, as well as at 1-week and 1-month follow-up.

Ethics and dissemination This protocol has been approved by the ethics committee of Yueyang Hospital of Integrated Traditional Chinese and Western Medicine (no. 2019-17). Written informed consent will be obtained from all participants. The results will be disseminated through peer-reviewed journals for publications.

Trial registration number ChiCTR1900023473; Preresults.

\section{INTRODUCTION}

In recent years, the incidence of chronic insomnia in adults has become higher, ${ }^{1}$ and short sleepers are also showing an increasing trend. ${ }^{2}$ Studies have found that patients with insomnia accompanied by short sleep duration can cause many chronic diseases, such

\section{Strengths and limitations of this study}

A randomised, single-centre, placebo acupuncture controlled trial with a $2 \times 2$ factorial design.

- The first trial to study the efficacy and safety of acupuncture for insomnia with short sleep duration.

- Participants will be screened for insomnia at baseline by polysomnography.

- Sleep indicators in the actigraphy will be used as objective outcomes of patients' sleep quality.

- One limitation is that this study is implemented in only one centre in Chinese subjects and without long-term treatment and follow-up.

as obesity, diabetes, cardiovascular disease, chronic kidney disease and hypertension, ${ }^{3-7}$ and may even result in inadequate hydration. ${ }^{8}$ Similarly, patients with insomnia with short sleep duration will have many adverse effects on their health. Some researchers have found that the mortality rate of patients with insomnia is three times more than that of people without insomnia. When patients with insomnia have short sleep duration, the damage to their health will be more serious and the mortality rate will be higher. ${ }^{9}$

Based on this, Vgontzas and FernandezMendoza ${ }^{10}$ proposed that insomnia can be divided into two phenotypes based on objective sleep duration, with the threshold of the total sleep time (TST) being 6 hours. The first phenotype (short objective sleep duration) is characterised by physiological hyperarousal with numerous medical complications. The second phenotype (normal sleep duration) is characterised by cognitive-emotional disorders, accompanied by a process of cortical excitement and remitting course. Vgontzas also suggested that insomnia with short sleep duration may be less sensitive to cognitivebehavioural therapy for insomnia (CBT-I). Although many guidelines of sleep disorders recommend CBT-I as the primary treatment, ${ }^{11-13}$ Bathgate et al conducted a trial and 
concluded that insomnia with short sleep duration has a dull response to CBT-I. ${ }^{14}$ The short objective sleep duration phenotype has a dull response to CBT-I because of a behaviourally based approach aimed at decreasing cognitive-emotional arousal, altering unhealthy sleep-related behaviours and beliefs and changing sleep misperceptions. ${ }^{15}$ However, the short objective sleep duration phenotype is mainly associated with cortical, and physiological hyperarousal (ie, short sleep duration and activation of the stress system), and non-remitting course. ${ }^{10}$ Obviously, CBT-I does not completely solve the symptoms of this phenotype. But it will respond better to treatments that primarily aim at decreasing physiological hyperarousal (eg, cortisol (COR)) and increasing sleep duration, such as medication or other biological treatments. ${ }^{16}$

Because insomnia with short sleep duration has a dull response to CBT-I treatment, these patients prefer to take sleep medicine. However, there are many side effects to benzodiazepine receptor agonists, such as hangover effects, cognitive impairment and drug addiction. ${ }^{17} 18$ Therefore, many people with insomnia seek complementary medicine, such as acupuncture, especially in China. ${ }^{19}$ Acupuncture has been widely used in the treatment of insomnia, and studies have shown that it can improve sleep efficiency (SE), daytime functioning, psychological health and sleep quality of insomnia subjects. ${ }^{20}{ }^{21}$ Our previous research also showed that acupuncture has a good short-term effect on perimenopausal insomnia. ${ }^{22}$ An earlier Cochrane review showed that acupuncture can improve the sleep quality of insomnia subjects compared with untreated groups and placebo acupuncture groups. ${ }^{23}$

We hypothesised that individuals with insomnia and short sleep duration would have a better treatment response to acupuncture than individuals with insomnia and normal sleep duration. We made this prediction because some studies have found that insomnia with objective short sleep duration is associated with activation of the stress system, especially the activation of the hypothalamic pituitary adrenal (HPA) axis, and the group with an objective short sleep duration had a higher amount of COR compared with the group with normal sleep duration. ${ }^{16} 2425$ Previous studies about acupuncture have shown that acupuncture can regulate the activity of the HPA axis and reduce adrenocorticotropic hormone, corticotrophin-releasing hormone and COR levels in peripheral blood. ${ }^{2627}$

According to the theory of traditional Chinese medicine, the main causes of insomnia are Yin deficiency leading to excessive fire, incoordination between the heart and the kidney, disturbance of heart due to phlegm heat, deficiency of both heart and spleen and liver depression forming fire. ${ }^{28}$ Therefore, we chose acupoints based on disease differentiation and special acupoints combinations to nourish Yin and drain fire, calm the mind and regulate mentality. SP 6 (Sanyinjiao) and HT 7 (Shenmen) are adopted as the main points to nourish Yin and drain fire, especially used to nourish liver and kidney Yin and decrease heart fire. ${ }^{29}$ PC 6 (Neiguan) is the collateral point of the hand-jueyin pericardium meridian, which is also specific acupuncture point of the eight confluent points. It is used to cool pericardium and restore consciousness. ${ }^{30}$ We also use GV20 (Baihui) and Ex-HN 1 (Sishencong) to make the brain activating and mind tranquillising. ${ }^{31}$

This is the first rigorous randomised controlled clinical trial to study the therapeutic effect of acupuncture on insomnia with short sleep duration and compare the effects on different insomnia phenotypes. Our objectives are as follows: (1) in randomised controlled trials with a $2 \times 2$ factorial design, we will study whether the clinical effect of acupuncture on chronic insomnia is related to insomnia phenotypes or acupoint effects and will evaluate both subjective and objective parameters. (2) We will explore the clinical symptomatic manifestations (difficulty in falling asleep, early awakening, difficulty in maintaining sleep, etc) and psychological characteristics (anxiety, depression, sensitivity, etc) and analyse the acupuncture effect for these symptoms of insomnia with short sleep duration.

\section{METHODS}

\section{Study design}

This study is a randomised controlled trial with a singlecentre, single-blind, $2 \times 2$ factorial design and will be completed in Yueyang Hospital of Integrated Traditional Chinese and Western Medicine affiliated with Shanghai University of Traditional Chinese Medicine. The study period will be from November 2019 to April 2021, and the Shanghai Municipal Commission of Health and Family Planning will be the management organisation of the study.

Insomnia subjects who meet the inclusion criteria will be divided into two groups according to the objective TST: insomnia with a short sleep duration group (objective sleep time $<6$ hours) and insomnia with a normal sleep duration group (objective sleep time $\geq 6$ hours). A total of 76 subjects will be recruited from each group. Each group will be randomly assigned to the acupuncture subgroup and the placebo acupuncture subgroup at a 1:1 ratio. The aim of this study is to evaluate the short-term efficacy of acupuncture for the treatment of insomnia with short sleep duration. According to our previous research, each subject will experience screening, treatment and a follow-up period of approximately 8 weeks.

All subjects will complete the following scales $^{32}$ : The Pittsburgh Sleep Quality Index (PSQI) ${ }^{33}$ and the Insomnia Severity Index (ISI) ${ }^{34} 35$ will evaluate subjects' subjective sleep improvement. Subjects will be assessed for the objective TST by actigraphy (ACT) ${ }^{36}$ in conjunction with a sleep diary. ${ }^{37}$ Subjects' mood improvement will be evaluated by the Beck Anxiety Inventory (BAI ${ }^{38}$ and the Beck Depression Inventory (BDI). ${ }^{39}$ The Fatigue Severity Scale (FSS) ${ }^{40}$ will be used to assess the improvement in fatigue. The above scales or ACT will be evaluated during the screening period and after the treatment 


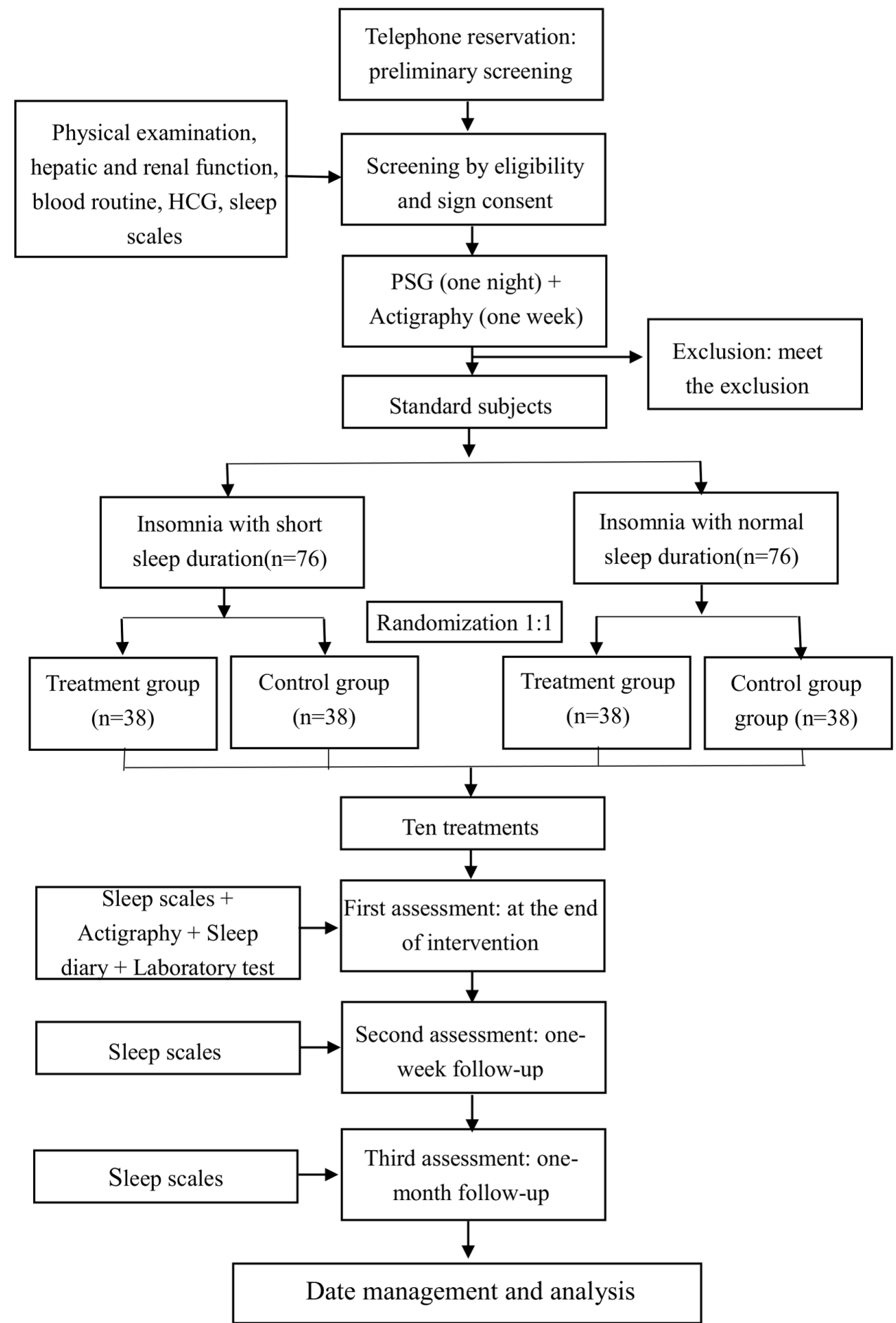

Figure 1 Trial flow chart. HCG, human chorionic gonadotropin; PSG, polysomnography.

at 1-week and 1-month follow-ups (figure 1). Polysomnography (PSG; Nihon Kohden, Japan) in this study will be used for screening purposes only. Subjects will sign the informed consent form after enrolment.

\section{Participants}

The study will include 152 participants with insomnia. We plan to recruit participants in multiple ways, and the recruitment location will be restricted to Yueyang Hospital of Integrated Traditional Chinese and Western Medicine affiliated with Shanghai University of Traditional Chinese Medicine, Shanghai, China. Our hospital is a comprehensive tertiary hospital with clinical treatment, scientific research and teaching practice. The main source of the subjects will be the outpatients who meet our criteria. In addition, we will also recruit participants through newspapers, social media and advertising. The time limit will be from November 2019 to April 2021. The site of our clinical trial will be the Insomnia Diagnosis and Treatment 
Center of the Acupuncture and Moxibustion Department at Yueyang Hospital of Integrated Traditional Chinese and Western Medicine affiliated with Shanghai University of Traditional Chinese Medicine. All participants who are willing to participate in the trial will be required to call our research coordinator and arrange an outpatient interview on the first available date.

\section{Inclusion criteria}

The outpatient interview will be conducted in the form of a semistructured interview questionnaire, which will contain the cause of insomnia, onset process, treatment process, main sleep problems, bedtime status, sleep-wake cycle, other sleep-related symptoms, daytime symptoms, mental state, and so on. ${ }^{41}$ The inclusion criteria have been developed based on the diagnostic criteria for chronic insomnia in the International Classification of Sleep Disorders (Third Edition) (ICSD-3) ${ }^{42}$ developed by the American Academy of Sleep Medicine in 2014. The inclusion criteria are as follows:

1. Between 18 and 65 years of age.

2. Compliance with the diagnostic criteria for chronic insomnia in ICSD-3.

3. Stable administration of sedative-hypnotic drugs for more than 3 months before entering the study or not taking such drugs.

4. Meet all of the following: $\mathrm{PSQI}^{33}>5$ points, $\mathrm{ISI}^{34}{ }^{35}>14$ points, $\mathrm{BAI}^{38}<45$ points and $\mathrm{BDI}^{39} \leq 28$ points.

5 . Never received acupuncture treatment.

6 . Voluntarily participated in the study and signed informed consent.

In addition, all participants will complete a 1-week sleep ACT and diary to determine their sleep patterns and objective sleep duration before enrolment. ${ }^{43}$ We will recruit 76 subjects in each group (insomnia with short sleep duration and insomnia with normal sleep duration). If one group completes recruitment first, that specific group will stop recruiting.

\section{Exclusion criteria}

The exclusion criteria are as follows:

1. Severe hepatic and renal function damage, as well as haematological diseases and respiratory diseases and diagnosis of mental disorders according to the Diagnostic and Statistical Manual of Mental Disorders $(\mathrm{DSM}-\mathrm{V}){ }^{44}$

2. Semistructured clinical interviews determine that subjects have other sleep disorders rather than primary insomnia.

3. Infectious diseases such as infectious hepatitis, tuberculosis, AIDS and syphilis.

4. Severe digestive system diseases and severe malnutrition.

5. Pregnant or lactating.

6. Severe trauma with no cure or not suitable for acupuncture, such as allergic constitution and severe dermatosis.
7. An Apnoea-Hypopnea Index $>10$ or periodic limb movements index during sleep associated with $>15$ arousals per hour on diagnostic PSG. ${ }^{45}$

8. Participated in other clinical trials in the last 3 months.

\section{Interventions}

The acupuncturist for this study has received a master's degree in acupuncture and tuina at Shanghai University of Traditional Chinese Medicine, has 8 years of training experience, has obtained a doctor qualification certificate and has 3 years of clinical work experience. All study participants will receive 10 days of training prior to the start of the trial to become more familiar with the process.

The subjects in the acupuncture group will be placed in the supine position. We have selected five acupoints for this study: GV20 (Baihui), PC 6 (Neiguan), SP 6 (Sanyinjiao), HT 7 (Shenmen) and Ex-HN 1 (Sishencong). The reason for choosing these acupoints is the previous systematic review ${ }^{46} 47$ and our clinical experience. A disposable sterile stainless steel acupuncture needle with a length of $40 \mathrm{~mm}$ and a diameter of $0.25 \mathrm{~mm}$ will be used (Andy, Guizhou, China). The acupuncturist will take the points on both sides of the body. The needle will be inserted into the skin to a depth of $10-20 \mathrm{~mm}$, which is determined by the acupuncturist according to body type (high or short, fat or thin). After piercing, a thrusting and twirling of the needle will be performed to induce the sensation of 'De qi', and the needle will be left for $20 \mathrm{~min} .{ }^{48}$ De qi means that after the needle has penetrated into the acupoint to a certain depth, the needle is thrust and twirled to cause the acupoint to obtain the induction of meridian qi. The subjects will have a self-conscious reaction such as soreness, heaviness or distention. This is the key process in acupuncture treatment. ${ }^{49}$

Subjects who entered the control group will receive a placebo acupuncture treatment by using a non-invasive placebo needle ${ }^{50}$ with the same acupoints as the treatment group. The Streitberger needle has been widely used in clinical research on placebo acupuncture treatment of insomnia, ${ }^{225152}$ and studies have shown that using this needle in a placebo acupuncture group is reliable for the Chinese population. ${ }^{53}$ Since the Streitberger needles will be in place for $20 \mathrm{~min}$, they will need to be firmly affixed to the skin or scalp. We have modified the procedure by using surgical tape (or hairpins in hairy regions) to hold the needles in place. This enables the needles to be applied in hairy regions and different needling directions to be attempted. Such a method has been adopted by many other researchers. ${ }^{52}$

Subjects from both the treatment group and the control group will be treated equally by the physician to prevent them from perceiving the difference. They will be informed about the acupuncture as follows: 'In this study, different types of acupuncture treatment will be compared. One type is the usual acupuncture, and the other is associated with positive outcomes in previous clinical studies' ${ }^{54}$ And subjects in both groups will be treated 3 times per week for 10 times by the same acupuncturist. 


\section{Outcome measures}

\section{Primary outcome measures}

The PSQI consists of 19 self-evaluation items and 5 other evaluation items. The self-assessment items assess sleep quality, fall asleep time, TST, SE, concomitant symptoms, hypnotic drugs and daytime functions. The total score is 0-21 points. Higher scores indicate worse sleep quality and more severe sleep disorders. ${ }^{33}$ The PSQI is widely used in the clinic to assess sleep dysfunction, ${ }^{55}$ and it is more likely to assess an individual's sleep state on weekdays. ${ }^{56}$ A total score $>5$ indicates a need for clinical treatment. ${ }^{57}$ The Chinese version of the PSQI has a high degree of reliability and validity for the Chinese population and can be used as an effective tool for sleep screening and evaluation in clinical and scientific research. ${ }^{5859}$

The ISI mainly evaluates the insomnia nature, insomnia severity and effects of daytime function on patients. The scale is simple and easy to implement. It consists of 7 evaluation items with a total score of 0-28. The higher the score, the more serious the degree of insomnia. ${ }^{35}$ It is mainly used for the screening of patients with insomnia and the evaluation of a therapeutic effect in clinical studies. ${ }^{60}$ A total score of 8-14 indicate subclinical insomnia, and a total score $>14$ indicate clinical insomnia. A change score of -8.4 points is associated with moderate improvement as rated by an independent assessor after treatment in the clinical sample. ${ }^{3561}$ The Chinese version of the ISI is used in China's population assessment for insomnia with high reliability and validity. ${ }^{62}$

\section{Secondary outcome measures}

The secondary outcome measures consist of (1) changes in the number of awakenings (NOA), total wake time (TWT), TST and SE measured by ACT before and after treatment; (2) changes in BAI, BDI and FSS scores after treatment and during follow-up compared with before treatment; (3) changes in sleep onset latency, TWT, TST, SE and middle of the night wake after sleep onset from the sleep diary after treatment compared with those before treatment (table 1).

ACT evaluates the body state by measuring the body movement by wearing a motion sensor on the nondominant wrist. It can be used as one of the objective indicators for evaluating sleep-wake state. ${ }^{36}$ The motion watch 8 wrist ACT produced by CamNtech will be used in this study. Its acceleration sensitivity is $<0.01 \mathrm{~g}$, with $5 \mathrm{~s}$ as the analysis unit. The recorded sleep and activity data will be analysed by the corresponding MotionWare Software. The main parameters include sleep-wake parameters and rest-activity parameters. It can satisfactorily evaluate the four sleep indicators: NOAs, wake time after sleep onset, TST and SE percentage. ${ }^{63}$ Early studies have shown that ACT has a good fit to PSG in assessing sleep and wakefulness (Rs=0.52-0.71) and has a good sensitivity as an index of effect evaluation in insomnia treatment. ${ }^{64}$ In this study, we will use ACT combined with a sleep diary ${ }^{37}$ to record the sleep of the subjects for one continuous week.
Changes in the NOA, TWT, TST and SE will be measured before and after treatment.

The BAI, which was developed by Beck et al in $1988,{ }^{66}$ contains a total of 21 different anxiety symptom items. It is used to evaluate the anxiety state of a subject in the past week. The higher the score, the more serious the degree of anxiety. Generally, BAI $\geq 45$ is used as the criterion for positive anxiety. The scale is simple in content, easy in operation and clear in understanding. The Chinese version has good reliability and validity. It is a commonly used measurement tool for anxiety symptom assessment in the Chinese population. ${ }^{67}$

The BDI is based on the diagnostic criteria for depression in the fourth edition of the DSM-V. The first version was published in 1961 and revised to the current version by Beck et al. The scale has a total of 21 items that are used to evaluate the severity of a subject's depression over the past week. The higher the total score, the more severe the depression. A total score of 0-13 is known as no depression, 14-19 as mild depression, 20-28 as moderate depression and 29-63 as major depression. The Chinese version has been tested and proven to have good reliability and validity. ${ }^{68}$

The FSS ${ }^{40}$ was formulated by Krupp et al in 1989. The scale has a total of nine items and is simple and easy to perform. A higher score indicates more severe fatigue. In patients with insomnia, the FSS threshold is 5.5 points, and a high score represents the impaired daytime functional status of patients with insomnia. ${ }^{69}$ The Chinese version of this scale has been determined to have good reliability and validity. ${ }^{70}$

\section{Safety assessments}

Safety will be assessed by routine blood test, renal function test and liver function test. These indicators are detected during the period of screening and after the treatment. We will still count the events during the trial through a list of adverse events. We will specifically evaluate them during the assessment phase $^{71}$ (table 1). Adverse events will be defined as any adverse medical reactions that occurred from the time the subject signed the informed consent form to the time of the last follow-up, whether or not there is a causal relationship with the study treatment. Subjects are required to fill in the list of adverse events, which should record the time point, severity, measures taken, whether they are related to the treatment and prognosis. During the assessment phase, researchers will assess the possible relationship between adverse events and the study, as well as the combined medications. Adverse events include all adverse reactions that are definitely related to treatment, most likely related to treatment and likely related to treatment.

\section{Sample size}

According to previous literature, ${ }^{22}$ placebo acupuncture reduced the PSQI of chronic insomnia to 14.76 after treatment, and the SD was 3.35. We hypothesise that the true acupuncture in our study could reduce the PSQI score 
Table 1 Trial processes chart

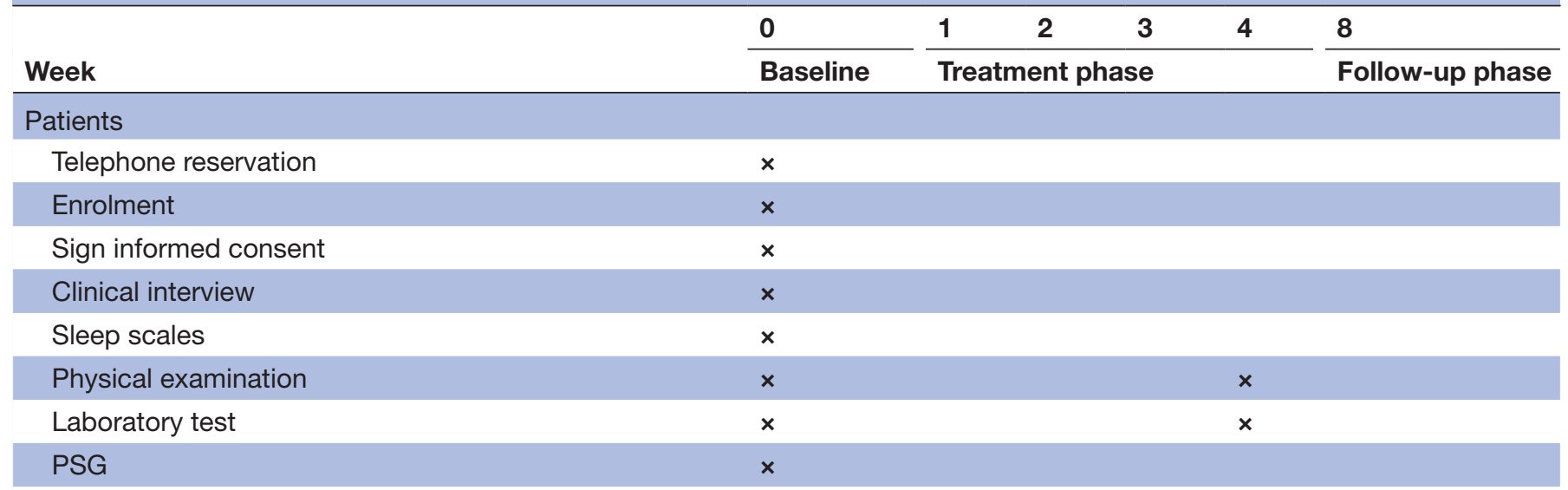

Groups

$\begin{array}{lr}\text { Acupuncture group (normal sleep duration) } & 10 \text { treatments } \\ \text { Control group (normal sleep duration) } & 10 \text { treatments } \\ \text { Acupuncture group (short sleep duration) } & 10 \text { treatments } \\ \text { Control group (short sleep duration) } & 10 \text { treatments }\end{array}$

Outcome measurement

\begin{tabular}{|c|c|c|c|c|c|c|}
\hline PSQI & $x$ & & & & $x$ & $x$ \\
\hline ISI & $x$ & & & & $x$ & $x$ \\
\hline ACT & $x$ & & & & $x$ & \\
\hline $\mathrm{BAl}$ & $x$ & & & & $x$ & \\
\hline BDI & $x$ & & & & $x$ & \\
\hline FSS & $x$ & & & & $x$ & \\
\hline Sleep diary & $x$ & $x$ & $x$ & $x$ & $x$ & \\
\hline Success of subject-blinding test & & & & & $x$ & \\
\hline \multicolumn{7}{|l|}{ Adverse events } \\
\hline Reasons for dropouts or withdrawals & & $x$ & $x$ & $x$ & $x$ & $x$ \\
\hline Patient's compliance & & & & & $x$ & $x$ \\
\hline
\end{tabular}

ACT, actigraphy; BAI, Beck Anxiety Inventory; BDI, Beck Depression Inventory; FSS, Fatigue Severity Scale; ISI, Insomnia Severity Index; PSG, polysomnography; PSQI, Pittsburgh Sleep Quality Index.

by 2.2 points compared with the placebo acupuncture, and the SD of 2.93 was taken from the previous research results. According to the needs of this study, we made $\alpha=0.05$ and $1-\beta=0.90$, according to the formula ${ }^{72}$ :

$$
n=\psi^{2}\left(\sum S_{i}^{2} / g\right) /\left[\sum\left(\bar{X}_{i}-\bar{X}\right)^{2} /(g-1)\right]
$$

$\bar{X}_{1}=12.56, S_{1}=2.93, \bar{X}_{2}=14.76, S_{2}=3.35, \bar{X}_{3}=12.56, S_{3}$ $=2.93, \bar{X}_{4}=14.76, S_{4}=3.35$ and $\bar{X}=13.66$; by looking up the table, $\psi=2.17$. Calculating the sample size of each group: $\mathrm{n}=30.92 \approx 31$; considering the loss factor (according to a $20 \%$ loss rate), the number of samples in each group is 38 cases, and the total number of samples in the 4 subgroups is 152 cases.

\section{Randomisation and blinding}

The statistician outside the study will use SPSS V.25.0 to generate two different sets of random numbers lists with
76 numbers in each. The random numbers in each list will be arranged in ascending order, with no. 1-38 for the control group and 39-76 for the acupuncture group. Subjects will enter the control group or the acupuncture group according to the random numbers corresponding to the order of signing the informed consent in a ratio of $1: 1$.

Random numbers and treatment protocols will be printed on the subject's treatment card and placed in an opaque envelope by an independent investigator. Each enrolled subject will be assigned to an order number and receive an envelope with their name on it. The acupuncturist will check whether the envelope was sealed and will open and remove the treatment card. During the trial, the generation of a random numbers list, subjects' recruitment, acupuncture treatment, outcome measures assessment and follow-up will be performed independently by different researchers. Only the outside assistant and the 
acupuncturist will be aware of the allocation. Participants and other relevant researchers will be blinded to the allocation.

The success of subject blinding will be assessed by, at the end of the last treatment session, asking the subject if they believe they are receiving active treatment.

\section{Data collection and management}

Data will be collected at the baseline (1 week before the first intervention), post intervention (at the end of intervention), 1-week follow-up and 1-month follow-up. Each visit will comprise three assessments: (1) subjects will complete sleep-related questionnaires independently in a private conference room at Yueyang Hospital of Integrated Traditional Chinese and Western Medicine, Shanghai University of Traditional Chinese Medicine. Completion of the questionnaires will require approximately $30 \mathrm{~min}$; during this time, outcomes assessors will be available to answer questions; (2) subjects will complete a sleep diary at home under the guidance of outcome assessors; (3) subjects will undergo one-night PSG at the baseline, ACT before and after the intervention. Outcome assessors will be trained to promote participant retention, collect good quality data and complete follow-up. Data analysts will be trained on data entry, coding, security and storage. Statisticians in the research team will provide training on data assessment and analysis. Maintenance of participant confidentiality will be involved: (1) asking subjects only share personal and study-related information during our study; (2) storing data in the password-protected files on a designated specific computer with restricted access; (3) only the research-related person have access to personal identifiable information, which will be destroyed once the study is completed. Technical appendix, statistical code and dataset available from the ResMan (www.medresman. org).

Subjects may withdraw from the study at any time for any reason. If any subject wishes to withdraw, the clinician will ask if they are willing to complete the final assessment and record the time of the last treatment. The incidence of withdrawal and loss to follow-up will be recorded and reported. We will also inquire the subjects about the reasons for absence, and will record compliance by the clinician.

\section{Statistics and analysis}

The statistics and analysis of all data will be performed by two analytical researchers independent of the trial. Intention-to-treat (ITT) and per-protocol (PP) analysis will be used as evaluation methods to evaluate the final results of this study. Among them, ITT analysis will be used as the main analytical method. The multiple imputation method will be the primary method for processing the missing data, and an observation carried forward method will also apply to sensitivity analysis. ${ }^{73}$ Data from dropout cases will be managed by both ITT analysis and PP analysis.
SPSS V.25.0 will be used for statistical analysis. For the measurement data that conform to the normal distribution and homogeneous variance, the mean $\pm \mathrm{SD}$ will be used to describe the discrete tendency and central tendency. The comparison between the two groups will be performed by independent samples t-test. The comparison of multiple time points will be based on repeated measurement data combined with multivariate analysis of variance. Measurement data that do not conform to the normal distribution will be described by median, minimum and maximum. The Mann-Whitney $\mathrm{U}$ rank sum test will be used for comparison between the two groups. The intragroup comparison will be based on the Friedman $(\mathrm{F})$ rank sum test of the relevant samples.

The enumeration data will be expressed by frequency and constituent ratio. If the analysis index is two-category and multicategory unordered data, the comparison between groups will be performed by $\chi^{2}$ test. If the analysis index is hierarchical order enumeration data, the Mann-Whitney U rank sum test will be used for the intragroup comparison.

All statistical tests will be performed on a two-sided test, and $p \leq 0.05$ will be considered statistically significant.

\section{DISCUSSION}

Some scholars have suggested that insomnia can be divided into two phenotypes based on objective sleep duration, namely, insomnia with short sleep duration and with normal sleep duration. The former is an important phenotype of insomnia and has more serious health hazards. ${ }^{3-9}$ At present, the treatment of insomnia tends to be biologically based (represented by benzodiazepine receptor agonists) and behaviourally based (represented by CBT-I). Most of the diagnosis and treatment guidelines of sleep disorders recommend CBT-I as the primary choice for treatment of insomnia and drug therapy as the secondary choice. ${ }^{1-13}$ However, studies have shown that patients with insomnia with a short sleep duration are not sensitive to CBT-I treatment, ${ }^{14}$ and these patients prefer to take sleep medicine. However, drug therapy has many adverse reactions, such as hangover effects, cognitive impairment and drug addiction. ${ }^{17} 18$ It is important to seek safe and effective complementary medicine with few side effects. This is the basis of our research. Acupuncture treatment for insomnia provides such an opportunity.

In this study, our first purpose is to determine whether acupuncture has a therapeutic effect on insomnia compared with the placebo acupuncture group. Second, we will determine whether acupuncture has a curative effect on insomnia with short sleep duration. Finally, we will examine whether acupuncture treatment for two phenotypes of insomnia has a difference in efficacy.

In fact, past studies have not completely found a reliable method to properly define the insomnia phenotype, which is largely due to the lack of biomarkers for insomnia or objective indicators that can be used for classification. Some clinical practice guidelines do not recommend 
PSG as a diagnostic tool and a severity assessment tool for insomnia. ${ }^{74}$ However, an increasing number of studies have shown that the use of objective sleep assessment tools to classify insomnia has a great effect on the analysis of many potential disease incidences. ${ }^{9} 1075$ This study provides further support for the use of an objective sleep index to help diagnose and treat the specific phenotypes of insomnia.

This study used ACT as a sleep time measurement tool and an objective assessment tool for sleep without introducing the PSG. Compared with ACT, PSG requires a bed rest time of approximately 8 hours, but ACT does not have this limitation. By reviewing the previous literature comparing the differences between the two measurement tools, ${ }^{64} 657677$ ACT has a good fit to PSG in assessing the time of sleep and wakefulness in patients with insomnia. However, to a certain extent, ACT is more likely to underestimate sleep maintenance time and overestimate the total arousal time, which may result in some of the subjects with a TST $>6$ hours being included in the group of patients with insomnia with short sleep duration. However, first, the gap is small. Second, the conclusions of these differences under this inclusion criterion may represent a greater actual difference between the two phenotypes. In addition, using one-night PSG to evaluate the subject's objective sleep time has a first-night effect. Therefore, we chose a week of ACT data to assess the objective indicators. Moreover, previous studies have shown that ACT can be used as a useful and efficient tool to assess the sleep patterns of individuals in their own sleep environment. ${ }^{7879}$

The use of placebo control in acupuncture trials remains controversial. ${ }^{80}$ In pharmacological treatment trials, an ideal placebo should be indistinguishable from the true interventions and be physiologically inert. ${ }^{81}$ However, it remains a challenge to design an adequate placebo for non-pharmacological interventions, such as acupuncture, in which non-specific treatment exists in the placebo group. ${ }^{82}$ Since placebo acupuncture can produce a significant non-specific therapeutic effects, there is little space left for the assumed specific effect of acupuncture. ${ }^{83}$ Therefore, we expect that in the future research work, researchers will invent a more authoritative placebo acupuncture.

This study is the first trial to use acupuncture as an intervention to treat different phenotypes of insomnia. Our conclusions will expand the research results of previous studies and will further explain that insomnia with short objective sleep duration is a biologically more serious insomnia phenotype. These patients require more specific attention and more specific treatment options.

\section{Patient and public involvement}

Patients' priorities, experience and preferences were not involved in the development of the research question and outcome measures, the design of this study, or the recruitment to and conduct of the study. The results will be not disseminated to study participants.
Correction notice The article has been corrected since it is published. The authorship has been updated and Xin-yu Chen has been added as an author in the author list.

Acknowledgements The authors thank Cong-quan Yin and Xiao-peng Ma for their comments and suggestions on the protocol.

Contributors $\mathrm{CW}$ and $\mathrm{Y}-\mathrm{fC}$ conceived the research plan. CW and W-jY drafted the protocol. X-tY, CF, J-jL and JW coordinated the study. W-IX, Y-xZ and CW recruited the subjects. CW and $\mathrm{W}-\mathrm{j} Y$ formed the analysis plan. $\mathrm{X}-\mathrm{yC}$ assisted the subject in completing the questionnaire.All authors participated in, read and approved the final manuscript.

Funding This work was supported by: The Construction of Chinese Medicine Heritage Innovation Platform (Project No.: ZY (2018-2020)-CCCX-2004-03); The Shanghai Science and Technology Commission (Project No.: 18401971000); The Shanghai Municipal Commission of Health and Family Planning (Project No.: ZYKC201703006); The National Natural Science Foundation of China (Grant No. 81503631); The Interdisciplinary Project of "Clinical Immunology of Traditional Chinese Medicine" in Shanghai (Project No.: 30304113598); The National Natural Science Foundation of China (Grant No. 81904300); The National Natural Science Foundation of China (Grant No. 81804200).

Disclaimer This funding source had no role in the design of this study and will not have any role during its execution, analyses, interpretation of the data, or decision to submit results.

\section{Competing interests None declared.}

Patient consent for publication Not required.

Ethics approval The trial has been approved by the ethics committee of Yueyang Hospital of Integrated Traditional Chinese and Western Medicine (No.: 2019-17).

Provenance and peer review Not commissioned; externally peer reviewed.

Open access This is an open access article distributed in accordance with the Creative Commons Attribution Non Commercial (CC BY-NC 4.0) license, which permits others to distribute, remix, adapt, build upon this work non-commercially, and license their derivative works on different terms, provided the original work is properly cited, appropriate credit is given, any changes made indicated, and the use is non-commercial. See: http://creativecommons.org/licenses/by-nc/4.0/.

ORCID iD

Cong Wang http://orcid.org/0000-0001-5000-2854

\section{REFERENCES}

1 Suh S, Cho N, Zhang J. Sex differences in insomnia: from epidemiology and etiology to intervention. Curr Psychiatry Rep 2018;20:69.

2 Knutson KL, Van Cauter E, Rathouz PJ, et al. Trends in the prevalence of short sleepers in the USA: 1975-2006. Sleep 2010;33:37-45.

3 Dashti HS, Scheer FA, Jacques PF, et al. Short sleep duration and dietary intake: epidemiologic evidence, mechanisms, and health implications. Adv Nutr 2015;6:648-59.

4 Ayas NT, White DP, Al-Delaimy WK, et al. A prospective study of selfreported sleep duration and incident diabetes in women. Diabetes Care 2003;26:380-4.

5 Tobaldini E, Fiorelli EM, Solbiati M, et al. Short sleep duration and cardiometabolic risk: from pathophysiology to clinical evidence. Nat Rev Cardiol 2018.

6 Li J, Huang Z, Hou J, et al. Sleep and CKD in Chinese adults: a cross-sectional study. Clin J Am Soc Nephrol 2017;12:885-92.

7 Vgontzas AN, Liao D, Bixler EO, et al. Insomnia with objective short sleep duration is associated with a high risk for hypertension. Sleep 2009;32:491-7.

8 Rosinger AY, Chang A-M, Buxton OM, et al. Short sleep duration is associated with inadequate hydration: cross-cultural evidence from US and Chinese adults. Sleep 2019;42. doi:10.1093/sleep/zsy210. [Epub ahead of print: 01 Feb 2019].

9 Sivertsen B, Pallesen S, Glozier N, et al. Midlife insomnia and subsequent mortality: the Hordaland health study. BMC Public Health 2014;14:720.

10 Vgontzas AN, Fernandez-Mendoza J. Insomnia with short sleep duration: nosological, diagnostic, and treatment implications. Sleep Med Clin 2013;8:309-22.

11 Qaseem A, Kansagara D, Forciea MA, et al. Management of chronic insomnia disorder in adults: a clinical practice guideline from the American College of physicians. Ann Intern Med 2016;165:125-33. 
12 Riemann D, Baglioni C, Bassetti C, et al. European guideline for the diagnosis and treatment of insomnia. J Sleep Res 2017;26:675-700.

13 Chinese Sleep Research Society. Guidelines for diagnosis and treatment of insomnia in China. Natl Med J China 2017;97:1844-56.

14 Bathgate CJ, Edinger JD, Krystal AD. Insomnia patients with objective short sleep duration have a blunted response to cognitive behavioral therapy for insomnia. Sleep 2017;40. doi:10.1093/sleep/ zsw012. [Epub ahead of print: 01 Jan 2017].

15 Trauer JM, Qian MY, Doyle JS, et al. Cognitive behavioral therapy for chronic insomnia: a systematic review and meta-analysis. Ann Intern Med 2015;163:191-204

16 Vgontzas AN, Fernandez-Mendoza J, Liao D, et al. Insomnia with objective short sleep duration: the most biologically severe phenotype of the disorder. Sleep Med Rev 2013;17:241-54.

17 Buscemi N, Vandermeer B, Friesen C, et al. The efficacy and safety of drug treatments for chronic insomnia in adults: a meta-analysis of RCTs. J Gen Intern Med 2007;22:1335-50.

18 Miyata A, Iwamoto K, Kawano N, et al. The effects of acute treatment with ramelteon, triazolam, and placebo on driving performance, cognitive function, and equilibrium function in healthy volunteers. Psychopharmacology 2015;232:2127-37.

19 China Sleep Research Association. Guidelines for the diagnosis and treatment of insomnia in China. National Medical Journal of China 2017;97:1844-56.

20 Sarris J, Byrne GJ. A systematic review of insomnia and complementary medicine. Sleep Med Rev 2011;15:99-106.

21 Yin X, Gou M, Xu J, et al. Efficacy and safety of acupuncture treatment on primary insomnia: a randomized controlled trial. Sleep Med 2017;37:193-200.

22 Fu C, Zhao N, Liu Z, et al. Acupuncture improves Peri-menopausal insomnia: a randomized controlled trial. Sleep 2017;40. doi:10.1093/ sleep/zsx153. [Epub ahead of print: 01 Nov 2017].

23 Cheuk DK, Yeung WF, Chung KF, et al. Acupuncture for insomnia. Cochrane Database Syst Rev 2012;9:CD005472.

24 Vgontzas AN, Tsigos C, Bixler EO, et al. Chronic insomnia and activity of the stress system: a preliminary study. J Psychosom Res 1998;45:21-31.

25 Vgontzas AN, Bixler EO, Lin HM, et al. Chronic insomnia is associated with nyctohemeral activation of the hypothalamicpituitary-adrenal axis: clinical implications. J Clin Endocrinol Metab 2001;86:3787-94.

26 Wei Y, Dong M, Zhong L, et al. Regulation of hypothalamic-pituitaryadrenal axis activity and immunologic function contributed to the anti-inflammatory effect of acupuncture in the OVA-induced murine asthma model. Neurosci Lett 2017;636:177-83.

27 Xue-fen WU, Xue-na Z, Xin GUO, et al. Research Progress of HPA - related Hormones Improving Sleep by Acupuncture Intervention. JCAM 2017;33:69-71.

28 Chen JM, Theory ZX. Traditional Chinese medicine. Shanghai: Shanghai Medical University, 1985.

29 Feng S-W, Zeng F, Ren Y-L, et al. [Discussion on disease spectrum treated with acupuncture at shenmen (HT 7) and its compatibility based on bibliometrics]. Zhongguo Zhen Jiu 2014;34:561-4.

30 Kim YS, Lee SH, Jung WS, et al. Intradermal acupuncture on shenmen and nei-kuan acupoints in patients with insomnia after stroke. Am J Chin Med 2004;32:771-8.

31 Guerreiro da Silva JB, Nakamura MU, Cordeiro JA, et al Acupuncture for low back pain in pregnancy--a prospective, quasirandomised, controlled study. Acupunct Med 2004;22:60-7.

32 Buysse DJ, Ancoli-Israel S, Edinger JD, et al. Recommendations for a standard research assessment of insomnia. Sleep 2006;29:1155-73.

33 Buysse DJ, Reynolds CF, Monk TH, et al. The Pittsburgh sleep quality index (PSQI): a new instrument for psychiatric research and practice. Psychiatry Res 1989;28:193-213.

34 Morin CM. Insomnia: psychological assessment and management. New York-London: The Guilford Press, 1993.

35 Bastien $\mathrm{CH}$, Vallières $\mathrm{A}$, Morin $\mathrm{CM}$. Validation of the insomnia severity index as an outcome measure for insomnia research. Sleep Med 2001;2:297-307

36 Ancoli-Israel S. Actigraphy. In: Kryger MH, Roth T, Dement WC eds. Principles and practice of sleep medicine. fourth edition. Philadelphia, PA: Saunders, 2005: 1459-67.

37 Sateia MJ. Epidemiology, consequences, and evaluation of insomnia. In: Lee-Chiong TL, Sateia MJ, Carskadon MA eds. Sleep medicine. Philadelphia, PA: Hanley and Belfus, 2002: 151-60.

38 Manne S, Nereo N, DuHamel K, et al. Anxiety and depression in mothers of children undergoing bone marrow transplant: symptom prevalence and use of the Beck depression and Beck anxiety inventories as screening instruments. J Consult Clin Psychol 2001;69:1037-47.
39 Beck AT, Steer RA, Ball R, et al. Comparison of Beck depression inventories -la and -II in psychiatric outpatients. J Pers Assess 1996;67:588-97.

40 Krupp LB, LaRocca NG, Muir-Nash J, et al. The fatigue severity scale. Application to patients with multiple sclerosis and systemic lupus erythematosus. Arch Neurol 1989;46:1121-3.

41 Schutte-Rodin S, Broch L, Buysse D, et al. Clinical guideline for the evaluation and management of chronic insomnia in adults. J Clin Sleep Med 2008;4:487-504.

42 Sateia M. American Academy of Sleep Medicine. In: International classification of sleep disorders. 3rd edn. USA: American Academy of Sleep Medicine, 2014.

43 Ibáñez V, Silva J, Cauli O. A survey on sleep questionnaires and diaries. Sleep Med 2018;42:90-6.

44 APA. Diagnostic and statistical manual of mental disorders: DSM- V. Washington (DC): American Psychiatric Publishing, 2013.

45 Yeung W-F, Chung K-F, Zhang S-P, et al. Electroacupuncture for primary insomnia: a randomized controlled trial. Sleep 2009;32:1039-47.

46 Huang W, Kutner N, Bliwise DL. A systematic review of the effects of acupuncture in treating insomnia. Sleep Med Rev 2009;13:73-104.

47 Huang K, Liang S, Xu Y, et al. [Law of acupoint selection in acupuncture treatment for insomnia based on data mining method] Zhongguo Zhen Jiu 2015;35:960-3.

48 White AR, Filshie J, Cummings TM. International acupuncture research forum. clinical trials of acupuncture: consensus recommendations for optimal treatment, sham controls and blinding. Complement Ther Med 2001;9:237-45.

49 Zhou W, Benharash P. Significance of "Deqi" response in acupuncture treatment: myth or reality. J Acupunct Meridian Stud 2014;7:186-9.

50 Streitberger K, Kleinhenz J. Introducing a placebo needle into acupuncture research. Lancet 1998;352:364-5.

51 Yeung W-F, Chung K-F, Tso K-C, et al. Electroacupuncture for residual insomnia associated with major depressive disorder: a randomized controlled trial. Sleep 2011;34:807-15.

$52 \mathrm{Kim} \mathrm{M}$, Kim J-E, Lee H-Y, et al. Electroacupuncture for treating insomnia in patients with cancer: a study protocol for a randomised pilot clinical trial. BMJ Open 2017;7:e016269.

53 Xie C-C, Wen X-Y, Jiang L, et al. Validity of the "streitberger" needle in a chinese population with acupuncture: a randomized, singleblinded, and crossover pilot study. Evid Based Complement Alternat Med 2013;2013:1-5.

54 Brinkhaus B, Witt CM, Jena S, et al. Interventions and physician characteristics in a randomized multicenter trial of acupuncture in patients with low-back pain. J Altern Complement Med 2006;12:649-57

55 Mollayeva T, Thurairajah P, Burton K, et al. The Pittsburgh sleep quality index as a screening tool for sleep dysfunction in clinical and non-clinical samples: a systematic review and meta-analysis. Sleep Med Rev 2016;25:52-73.

56 Pilz LK, Keller LK, Lenssen D, et al. Time to rethink sleep quality: PSQI scores reflect sleep quality on workdays. Sleep 2018;41. doi:10.1093/sleep/zsy029. [Epub ahead of print: 01 May 2018].

57 Backhaus J, Junghanns K, Broocks A, et al. Test-Retest reliability and validity of the Pittsburgh sleep quality index in primary insomnia. $J$ Psychosom Res 2002;53:737-40.

58 Zheng B, Li M, Wang KL, et al. [Analysis of the reliability and validity of the Chinese version of Pittsburgh sleep quality index among medical college students]. Beijing Da Xue Xue Bao Yi Xue Ban 2016:48:424-8

59 Liu XC, Tang MQ, Hu L, et al. Reliability and validity of the Pittsburgh sleep quality index. Chin J Psyehiatry 1996;29:103-7.

60 Chen P-Y, Yang C-M, Morin CM. Validating the cross-cultural factor structure and invariance property of the insomnia severity index: evidence based on ordinal EFA and CFA. Sleep Med 2015;16:598-603.

61 Morin CM, Belleville G, Bélanger L, et al. The insomnia severity index: psychometric indicators to detect insomnia cases and evaluate treatment response. Sleep 2011;34:601-8.

62 Yu DSF. Insomnia severity index: psychometric properties with Chinese community-dwelling older people. J Adv Nurs 2010;66:2350-9.

63 Lichstein KL, Stone KC, Donaldson J, et al. Actigraphy validation with insomnia. Sleep 2006;29:232-9.

64 Vallières A, Morin CM. Actigraphy in the assessment of insomnia Sleep 2003;26:902-6.

65 Natale V, Plazzi G, Martoni M. Actigraphy in the assessment of insomnia: a quantitative approach. Sleep 2009;32:767-71. 
66 Beck AT, Epstein N, Brown G, et al. An inventory for measuring clinical anxiety: psychometric properties. J Consult Clin Psychol 1988;56:893-7.

67 Ceng KW, Wong CW, Wong KC, et al. A study of psychometric properties, normative scores and factor structure of Beck anxiety inventory Chinese version. Chin J Clin Psychol 2002;10:4-6.

68 Wang Z, Yuan CM, Huang J, et al. Reliability and validity of the Chinese version of Beck depression Inventory-II among depression patients. Chinese Mental Health Journal 2011;25:476-80.

69 Lichstein KL, Durrence HH, Riedel BW, et al. Epidemiology of sleep: age, gender, and ethnicity. New Jersey: Lawrence Erlbaum Associates, Inc, 2004.

70 Wang M-Y, Liu I-C, Chiu C-H, et al. Cultural adaptation and validation of the Chinese version of the fatigue severity scale in patients with major depressive disorder and nondepressive people. Qual Life Res 2016;25:89-99.

71 Levine J, Schooler NR. SAFTEE: a technique for the systematic assessment of side effects in clinical trials. Psychopharmacol Bull 1986;22:343-81.

72 Sakpal TV. Sample size estimation in clinical trial. Perspect Clin Res 2010;1:67-9.

73 Chandan S. Jones MP bias in the last observation carried forward method under informative dropout. J Stat Plan Inference 2009;139:246-55.

74 Littner M, Hirshkowitz M, Kramer M, et al. Practice parameters for using polysomnography to evaluate insomnia: an update. Sleep 2003;26:754-60.
75 Fernandez-Mendoza J, Shea S, Vgontzas AN, et al. Insomnia and incident depression: role of objective sleep duration and natural history. J Sleep Res 2015;24:390-8.

76 Edinger JD, Means MK, Stechuchak KM, et al. A pilot study of inexpensive sleep-assessment devices. Behav Sleep Med 2004;2:41-9.

77 Friedman L, Benson K, Noda A, et al. An actigraphic comparison of sleep restriction and sleep hygiene treatments for insomnia in older adults. J Geriatr Psychiatry Neurol 2000;13:17-27.

78 Ancoli-Israel S, Cole R, Alessi C, et al. The role of actigraphy in the study of sleep and circadian rhythms. Sleep 2003;26:342-92.

79 Sánchez-Ortuño MM, Edinger JD, Means MK, et al. Home is where sleep is: an ecological approach to test the validity of actigraphy for the assessment of insomnia. J Clin Sleep Med 2010;6:21-9.

80 Walach $\mathrm{H}$. The efficacy paradox in randomized controlled trials of CAM and elsewhere: beware of the placebo trap. $J$ Altern Complement Med 2001;7:213-8.

81 Paterson C, Dieppe P. Characteristic and incidental (placebo) effects in complex interventions such as acupuncture. BMJ 2005;330:1202-5.

82 Verhoef MJ, Lewith G, Ritenbaugh C, et al. Complementary and alternative medicine whole systems research: beyond identification of inadequacies of the RCT. Complement Ther Med 2005;13:206-12.

83 Zheng Y-C, Yuan T-T, Liu T. Is acupuncture a placebo therapy? Complement Ther Med 2014;22:724-30. 
Correction: Acupuncture for insomnia with short sleep duration: protocol for a randomised controlled trial

Wang C, Yang W, Yu X, et al. Acupuncture for insomnia with short sleep duration: protocol for a randomised controlled trial. BMJ Open 2020;10:e033731. doi: 10.1136/ bmjopen-2019-033731

This article was previously published with an error in authorship.

'Xin-yu Chen' has been added as the 9th author before 'Yun-fei Chen' in the revised version. The updated authorship is below

Wang, ${ }^{1}$ Wen-jia Yang, ${ }^{1}$ Xin-tong Yu, ${ }^{2}$ Cong Fu, ${ }^{1}$ Jin-jin Li, ${ }^{1}$ Jing Wang, ${ }^{1}$ Wen-lin Xu, ${ }^{1}$ Yi-xin Zheng, ${ }^{1}$ Xin-yu Chen, ${ }^{3}$ Yun-fei Chen ${ }^{1}$

Open access This is an open access article distributed in accordance with the Creative Commons Attribution Non Commercial (CC BY-NC 4.0) license, which permits others to distribute, remix, adapt, build upon this work non-commercially, and license their derivative works on different terms, provided the original work is properly cited, appropriate credit is given, any changes made indicated, and the use is non-commercial. See: http://creativecommons.org/licenses/by-nc/4.0/.

@ Author(s) (or their employer(s)) 2020. No commercial re-use. See rights and permissions. Published by BMJ.

BMJ Open 2020;10:e033731corr1. doi:10.1136/bmjopen-2019-033731corr1 